\title{
Modelo de Programación No Lineal para el Diseño de Sistemas de Tratamiento Electroquímico de Aguas Residuales Contaminadas con Trazas de Amoxicilina
}

\author{
Adrián López-Yáñez ${ }^{(1)}$, Juan M. Zamora-Mata(2), Alejandro Alonso ${ }^{(3)}$ y Jorge Ramírez-Muñoz ${ }^{(1)}$ \\ (1) Departamento de Energía, Universidad Autónoma Metropolitana-Azcapotzalco, Av. San Pablo No. 180, \\ Col. Reynosa Tamaulipas, Azcapotzalco, 02200, Ciudad de México, México. \\ (2) Departamento de Ingeniería de Procesos e Hidráulica, Universidad Autónoma Metropolitana-Iztapalapa, \\ Av. San Rafael Atlixco No. 186, Col. Vicentina, Iztapalapa, 09340, Ciudad de México, México. \\ (3) CONACYT-Universidad Autónoma Metropolitana-Azcapotzalco. Av. San Pablo 180, Col. Reynosa \\ Tamaulipas, Azcapotzalco, 02200, Ciudad de México, México. \\ (e-mail:mixtili_5050@hotmail.com;jmzm@xanum.uam.mx; iq_alonso@yahoo.com.mx, \\ jrm@correo.azc.uam.mx)
}

Recibido Nov. 13, 2015; Aceptado Ene. 14, 2016; Versión final Feb. 25, 2016, Publicado Oct. 2016

\begin{abstract}
Resumen
En este trabajo, se formula un modelo de programación no lineal (PNL) para el diseño de sistemas de tratamiento selectivo de efluentes que contienen trazas de amoxicilina. La presencia de antibióticos en aguas superficiales o subterráneas representa un problema ambiental importante por su potencial bioacumulativo y el desarrollo de resistencia antibiótica. Los métodos para la eliminación de fármacos y antibióticos incluyen la oxidación química usando reactivos Fenton, floculación y fotoquímica. Sin embargo, sus eficiencias de remoción son limitadas y se requiere de estudios orientados a optimizar los costos de tratamiento. Para la degradación de la amoxicilina se incorpora en el sistema una tecnología electroquímica. El modelo de PNL desarrollado se construye con base en una superestructura básica de red que expande el espacio de alternativas posibles de tratamiento, minimizando el flujo total de efluente que es tratado. Se resuelven cuatro ejemplos para mostrar la versatilidad y la utilidad del modelo propuesto.
\end{abstract}

Palabras clave: degradación de amoxicilina; tratamiento de aguas residuales; coagulación electroquímica; diseño óptimo

\section{Nonlinear Programming Model for the Design of Electrochemical Treatment Systems for Wastewater Streams Contaminated with Amoxicillin Traces}

\begin{abstract}
In this work, a nonlinear programming (NLP) model is formulated for the design of selective effluent treatment systems containing traces of amoxicillin. The presence of antibiotic in surface water bodies or groundwater generates an important environmental problem due to its bio-accumulative potential and the development of antibiotic resilient bacteria. Current methods employed in the removal of drugs and antibiotics include chemical oxidation by Fenton reagents, flocculation and photochemical treatments. However, their removal efficiencies are still limited and studies are required for the optimization of the treatment costs. An electrochemical technology is incorporated in the system for the degradation of amoxicillin. The NLP model developed is built from a basic network superstructure that expands the space of alternative treatment possibilities, minimizing the total flowrate that is sent to treatment. Four illustrative examples are solved to show the versatility and usefulness of the model proposed.
\end{abstract}

Keywords: amoxicillin degradation; wastewater treatment; electrochemical coagulation; optimal design. 


\section{INTRODUCCIÓN}

Desde la Segunda Guerra Mundial se han producido y utilizado grandes cantidades de antibióticos y fármacos, con el fin de prevenir enfermedades o mejorar la salud de la población (Homem et al., 2010). Sin embargo, su presencia en mantos acuíferos o aguas subterráneas, provoca una contaminación ambiental grave, lo cual ha generado recientemente gran preocupación no sólo por su alto potencial bio-acumulativo, sino también debido a la aparición de bacterias resistentes a los antibióticos. La presencia de este tipo de agentes en el medio ambiente, puede afectar a los animales con órganos, tejidos, células o biomoléculas similares a las del ser humano (Fent et al., 2006). Los antibióticos, desechos farmacéuticos y otros productos químicos no regulados, al ser desechados e incorporados al ambiente son comúnmente denominados contaminantes emergentes. La regulación, tratamiento y emisión de estas sustancias han recibido poca atención en la mayoría de los países del mundo. Su inclusión como residuos peligrosos depende del daño a la salud humana, así como de su presencia y capacidad de dispersión en los recursos hídricos (Petrovic et al., 2003). Los antibióticos se encuentran entre los medicamentos que más se venden y se consumen en México. Éstos representaron en el 2005 un mercado anual de 960 millones de dólares, una proporción mayor si se compara con otros países desarrollados (Dreser et al., 2008). De acuerdo con la Asociación Nacional de Farmacias de México, los antibióticos más vendidos en México son: antibacterianos sistemáticos, penicilina y sus derivados, cefalosporinas, trimetropina y agentes similares, y macrólidos y similares (García, 2010).

La amoxicilina es un antibiótico derivado de la penicilina y es ampliamente utilizado para tratar una gran cantidad de infecciones causadas por bacterias. Su metabolismo natural dentro del cuerpo humano hace que su concentración en excreciones alcance niveles importantes, sobre todo en los efluentes de las unidades de cuidado de la salud, por ejemplo, aguas residuales hospitalarias. Esto conlleva a su presencia aún en muy bajas concentraciones en las aguas residuales y consecuentemente a su aparición en el medio ambiente. Por lo anterior, la eliminación de amoxicilina presente en aguas residuales se ha convertido en un problema de interés (Padilla et al., 2015).

Existen diversas tecnologías que se pueden emplear para la eliminación de contaminantes emergentes, tales como los procesos de oxidación avanzada que son utilizados en la remoción de amoxicilina presente en volúmenes de agua de desecho. Este tipo de tecnologías se caracteriza principalmente por sus altas tasas de degradación (Quesada et al., 2009), por lo que existe una gran cantidad de trabajos en la literatura que evalúan la eficiencia de este proceso. Una característica de esta tecnología es la formación de radicales hidroxilo $(\cdot \mathrm{OH})$ en altas concentraciones, los cuales tienen altos potenciales de oxidación que se pueden utilizar para romper algunos enlaces de moléculas orgánicas. Los procesos más usados de oxidación avanzada son: reacciones Fenton, fotocatálisis, ozonación, electrólisis, ultrasonido y oxidación húmeda catalítica de aire. La combinación de estos procesos ha sido comúnmente empleada para obtener mayores eficiencias en la degradación de los contaminantes emergentes, incluyendo la eliminación de amoxicilina (Quesada et al., 2009; Klavarioti et al., 2009).

Un proceso de electrocoagulación puede también ser usado para la eliminación de contaminantes farmacéuticos (Padilla et al., 2015). Este tipo de proceso requiere de un reactor electroquímico, en donde un ánodo de sacrificio se disuelve liberando iones inestables (Nidheesh y Gandhimathi, 2012; Arsand et al., 2013). Para comprender con mayor claridad el proceso de electrocoagulación, es posible partir del análisis de los sistemas coloidales, como es el caso que se presenta en la mayoría de las disoluciones de fármacos en agua. En estos sistemas, la fase dispersa se encuentra en unidades cuyo tamaño está entre 0.001 y 0.1 $\mu \mathrm{m}$, llamadas micelas. Las micelas establecen diversas relaciones con el medio dispersante para mantenerse en estado estable. Regularmente las fuerzas más importantes que permiten a las micelas su incorporación a la fase dispersante, son las producidas por las cargas electrostáticas. En la coagulación, se busca eliminar estas interacciones mediante la adición de un agente que les impida o limite, de esta forma a cualquier sustancia que logre este objetivo se le conoce como coagulante (Freitas et al., 2015).

Los coagulantes pueden ser adicionados desde una fuente externa a la solución, o bien encontrarse dentro del mismo sistema coloidal, siendo posible incluso que las propias micelas pierdan estabilidad debido a cambios en la tensión superficial, convirtiéndose en coagulantes, aunque este proceso suele ser generalmente lento (Xuedong et al., 2014). Por otro lado, ha sido práctica común la adición de ciertas sales de baja solubilidad como sulfatos, fosfatos o hidróxidos de aluminio y hierro principalmente. Estos componentes forman coágulos con la fase dispersa en tiempos relativamente cortos, aumentando su concentración hasta alcanzar valores críticos. Cuando esto sucede, comienzan a formarse flóculos insolubles que son generalmente hidrofóbicos. Los flóculos tienden a atrapar en su estructura algunos componentes insolubles presentes en solución como la amoxicilina $u$ otros contaminantes. La electrocoagulación evita la adición de sales insolubles, a cambio de su formación in situ mediante la oxidación de un ánodo de sacrificio, que regularmente está constituido de chatarra hierro o aluminio, con lo 
que los costos de operación pueden disminuir de forma considerable. Además, mediante el control del potencial de oxidación o de la corriente que pasa por el reactor electroquímico, es posible controlar la tasa a la cual se incorpora el coagulante hacia el interior del sistema coloidal, con el fin de mantener una tasa de remoción del contaminante en un valor constante (Xuedong et al., 2014). La coagulación y la electrocoagulación son particularmente útiles en la eliminación de contaminantes, ya que la formación de sólidos rompe la estabilidad coloidal. Por otra parte, debido a su naturaleza hidrofóbica, la eliminación mecánica de flóculos se puede lograr de una manera relativamente sencilla (Butler et al., 2011).

Padilla et al. (2015) estudiaron la eliminación de amoxicilina mediante un proceso de electrocoagulación, usando electrodos de trabajo de hierro y carbón vítreo. Estos autores reportaron una tasa máxima de remoción de alrededor del $80 \%$ para una celda electroquímica no optimizada. De acuerdo con sus resultados experimentales, el siguiente modelo cinético de orden variable se puede aplicar a un proceso de electrocoagulación para la eliminación de la amoxicilina como se muestra en la Ec.1:

$$
-r_{j}=-\frac{d C_{j}}{d t}=\frac{K_{1} C_{j}}{1+K_{2} C_{j}} .
$$

Donde $K_{1}$ y $K_{2}$ son constantes cinéticas cuyos valores fueron también reportados por Padilla et al. (2015) para los dos ánodos que se evaluaron, y $C_{j}$ representa la concentración de amoxicilina.

En las industrias productivas y hospitales, comúnmente se generan cantidades importantes de aguas residuales que deben ser tratadas para remover o degradar los diferentes contaminantes. El diseño de sistemas de tratamiento que involucran un solo contaminante y tasas de remoción constante puede ser abordado de manera eficiente con metodologías de punto de pliegue (e.g. Wang y Smith, 1994; Bagajewicz, 2000). También, el diseño de sistemas de tratamiento multi-contaminante con base a unidades de tratamiento caracterizadas por tasas de remoción constante, ha sido abordado en la literatura con métodos basados en técnicas de programación matemática. Dichos métodos se desarrollan a partir de superestructuras del sistema de tratamiento, que expanden un conjunto amplio de soluciones factibles (e.g. Galán y Grossmann, 1999; Grossmann et al., 1999; Zamora-Mata et al., 2004; Hernández-Suárez et al., 2004; Alva et al., 2007; Faria y Bagajewicz, 2009). A cada superestructura de red utilizada, se le asocia un modelo de PNL o programación no lineal entera-mixta (PNL-EM), cuya solución permite obtener diseños óptimos para las redes de tratamiento.

Por otra parte, la solución de algunos de los modelos de PNL utilizados representa un reto para la obtención de soluciones óptimas globales dado su carácter no convexo, que puede dar lugar a dificultades numéricas y múltiples óptimos locales. Diferentes autores se han dado a la tarea de desarrollar algoritmos que permitan obtener de manera sistemática la mejor solución óptima local posible. Por ejemplo, Galán y Grossmann (1999) proponen utilizar modelos de programación lineal obtenidos de la relajación de su modelo original de PNL. La solución de los modelos relajados de programación lineal busca producir buenos puntos de arranque para los modelos de PNL no convexos. Alva et al. (2007) proponen una metodología para la síntesis de sistemas integrados que incluyen operaciones usuarias de agua, tratamiento de efluentes, reciclo de agua tratada y distribución de agua fresca. El método de diseño propuesto utiliza elementos de la metodología de punto de pliegue con tasas de remoción constantes y un modelo de superestructura del tipo de PNL-EM, en donde cada operación usuaria de agua y la unidad de tratamiento son precedidos por un mezclador y seguidos por un divisor. Las fuentes de agua fresca se introducen con un divisor y las descargas de agua residual se dirigen hacia un mezclador final. Faria y Bagajewicz (2009) presentan una metodología para el diseño y la reconfiguración de sistemas usuarios de agua que incorporan regeneración con re-uso de agua. Para dicho propósito estos autores utilizan un modelo de PNLEM que maximiza el valor presente neto de la red o la tasa de retorno de la inversión, en lugar de minimizar el consumo de agua fresca. Sin embargo, para modelar los procesos de regeneración, estos autores suponen constante la concentración de al menos un contaminante a la salida de la unidad de tratamiento de agua para reúso.

Zamora-Mata et al. (2004) propusieron un modelo de programación lineal para diseñar un sistema de tratamiento selectivo de efluentes con base en la superestructura básica de la Fig. 1. Sin embargo, estos autores también suponen que la unidad de tratamiento opera con tasas de remoción constantes para los contaminantes involucrados. En el problema abordado por estos autores los flujos másicos de las corrientes $\left(S_{i}\right)$ y las concentraciones de contaminantes a la entrada $\left(C_{i, j}\right)$ se dan por conocidos. La solución del modelo de programación lineal determina los valores óptimos de las variables desconocidas de flujo $\left(t, f_{i}, f_{i, e}\right)$ y concentración $\left(\operatorname{cin}_{j}, \operatorname{cout}_{j}\right.$ y $\left.c_{j, e}\right)$, bajo la restricción de que la concentración de salida de cada contaminante $\left(c_{j, e}\right)$ debe estar por debajo del límite fijado por la norma ambiental de descarga de efluentes $\left(c_{j, e},\right)$.

En este trabajo se aborda el problema de diseño de sistemas de tratamiento selectivo de corrientes efluentes 
contaminadas con trazas de amoxicilina. Con este objetivo, se propone un modelo de programación no lineal que utiliza la superestructura básica de Zamora-Mata et al. (2004), con una unidad de tratamiento que opera con una tasa de remoción variable, basada en el proceso de electrocoagulación estudiado por Padilla et al. (2015), y modelado con la cinética de la Ec. (1). En la siguiente sección se presenta el modelo propuesto, posteriormente se resuelven cuatro ejemplos ilustrativos y se finaliza con la presentación de conclusiones.

\section{DESARROLLO DEL MODELO}

En el presente trabajo el problema se plantea de la siguiente manera: se cuenta con un conjunto de corrientes efluentes con caudales conocidos que contienen una concentración también conocida de amoxicilina (Tabla 1), se procesa mediante una tecnología de tratamiento electroquímico para remover el fármaco. En esta tecnología la concentración de salida de la amoxicilina depende de la concentración de entrada y se aplica un modelo cinético de orden variable, de acuerdo al trabajo presentado por Padilla et al. (2015), en el que se utilizó un ánodo de sacrificio de chatarra de hierro. Detalles adicionales del proceso de electrocoagulación pueden ser consultados en el trabajo de Padilla et al. (2015). El objetivo de este problema es la determinación de un diseño óptimo del sistema de tratamiento y sus correspondientes caudales que satisfagan las normas de descarga para el contaminante. Los costos totales se suponen proporcionales al flujo de tratamiento $t$, por tal motivo, en éste trabajo se minimiza el flujo de tratamiento.

La superestructura básica considerada para el diseño óptimo de red (Zamora-Mata et al., 2004), incluye divisores (S), mezcladores (M), una unidad de tratamiento (UT) y las interconexiones. La Fig. 1 muestra la superestructura de red que cubre todas las alternativas de tratamiento contempladas sin considerar recirculaciones. Se formuló un modelo de optimización asociado con dicha superestructura como un problema de programación no lineal. El modelo de PNL que se presenta a continuación consiste de una función objetivo que minimiza el caudal de tratamiento, balances de masa para las aguas residuales, balances de contaminante en los mezcladores y las relaciones de entrada-salida de la unidad de tratamiento.

Tabla 1: Datos para el proceso de tratamiento de los ejemplos presentados

\begin{tabular}{|c|c|c|c|}
\hline$I$ & $\begin{array}{c}S_{i} \\
\left(\mathrm{Lmin}^{-1}\right)\end{array}$ & $\begin{array}{c}C_{i, j} \\
\left(\mathrm{mgL}^{-1}\right)\end{array}$ & $m_{i, j}=S_{i} C_{i, j} \times 10^{-3}\left(\mathrm{~g} \mathrm{~min}^{-1}\right)$ \\
\hline 1 & 6 & 28 & 0.1680 \\
\hline 2 & 5.5 & 21 & 0.1155 \\
\hline 3 & 4.5 & 19 & 0.0855 \\
\hline 4 & 4 & 17 & 0.0680 \\
\hline 5 & 3 & 16 & 0.0480 \\
\hline \multicolumn{3}{|c|}{$\Delta m_{j}^{U}=\Sigma m_{i, j}$} & 0.4850 \\
\hline
\end{tabular}

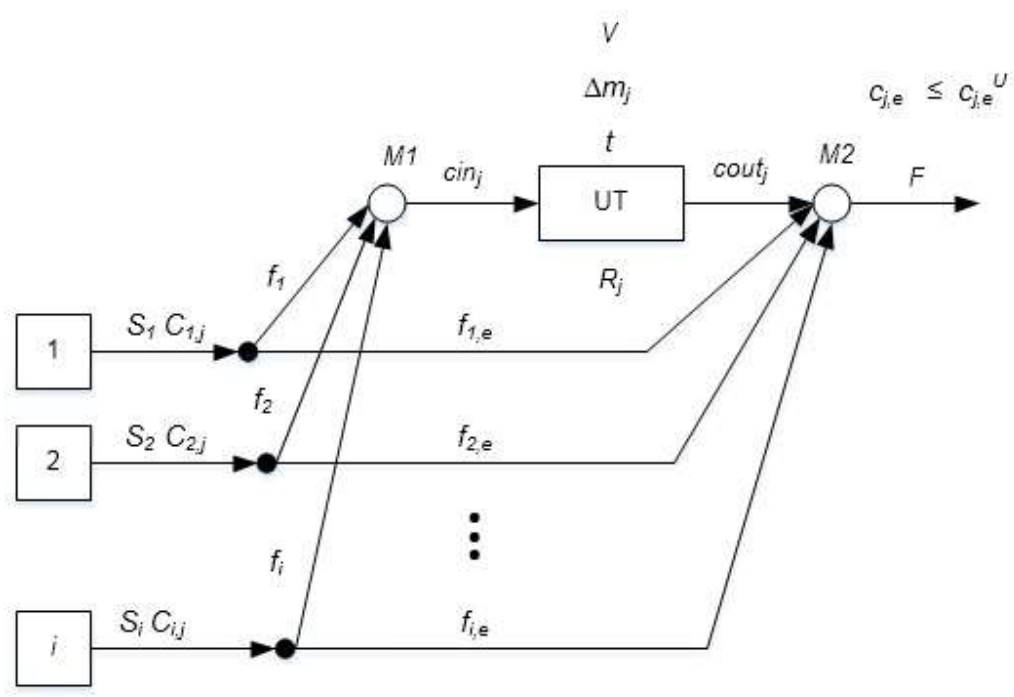

Fig. 1: Superestructura básica (adaptada de Zamora-Mata et al., 2004). 
Modelo de PNL.

Función objetivo:

Min $f(t)=t$

Sujeto a:

Balances de flujo de agua en los divisores:

$S_{1}=f_{1}+f_{1, e}$

$S_{2}=f_{2}+f_{2, e}$

$S_{3}=f_{3}+f_{3, e}$

$S_{4}=f_{4}+f_{4, e}$

$S_{5}=f_{5}+f_{5, e}$

Balance de flujo de agua residual en el mezclador antes de la unidad de tratamiento:

$t=f_{1}+f_{2}+f_{3}+f_{4}+f_{5}$

Balance de flujo de agua residual en el mezclador después de la unidad de tratamiento:

$t+f_{1, e}+f_{2, e}+f_{3, e}+f_{4, e}+f_{5, e}=F_{e}$

Carga másica de contaminante que es removida en la unidad de tratamiento:

$\Delta m_{j}=(0.001) t R_{j} \operatorname{cin}_{j}$

Balance de contaminante en el punto de descarga:

$F_{e} C_{j, e}=t$ cout $_{j}+f_{1, e} C_{1, j}+f_{2, e} C_{2, j}+f_{3, e} C_{3, j}+f_{4, e} C_{4, j}+f_{5, e} C_{5, j}$

Balance de contaminante en la entrada de la unidad de tratamiento:

$t \operatorname{cin}_{j}=f_{1} C_{1, j}+f_{2} C_{2, j}+f_{3} C_{3, j}+f_{4} C_{4, j}+f_{5} C_{5, j}$

Tasa de remoción variable de contaminante en la unidad de tratamiento:

$R_{j}=\frac{\operatorname{cin}_{j} \operatorname{cout}_{j}}{\operatorname{cin}_{j}}$

Tasa de electrocoagulación de amoxicilina usando ánodo de hierro:

$-r_{j}=\frac{K_{1} \text { cout }_{j}}{1+K_{2} \text { cout }_{j}}$

Balance de masa para la amoxicilina en el reactor electroquímico:

$-60 r_{j} V=t \operatorname{cin}_{j} R_{j}$

Cotas:

$f_{i}, f_{i, e}, t \leq F_{e} \quad i \in I$,

$c_{j, e} \leq c_{j, e}^{U}$ 


$$
\begin{aligned}
& \Delta m_{j} \leq \Delta m_{j}^{U} \\
& V^{L} \leq V \leq V^{U} \\
& 0 \leq R_{j} \leq 1
\end{aligned}
$$

El modelo de PNL desarrollado se optimiza utilizando un código de programación implementado en el sistema GAMS y el programa utilizado es MINOS (Brooke et al., 2012).

\section{RESULTADOS Y DISCUSIÓN}

El modelo de PNL se explica con la solución de cuatro ejemplos ilustrativos, esto con el fin de observar el desempeño del sistema de tratamiento al variar los parámetros de volumen del reactor y de concentración de amoxicilina en la descarga. Dado que la amoxicilina es un contaminante emergente y no existe una regulación sobre las concentraciones límites en las corrientes de descarga, por tal motivo, en los Ejemplos 1 y 2 la concentración de descarga de amoxicilina se fijó arbitrariamente en 12 y $10 \mathrm{mg} \mathrm{L}^{-1}$, respectivamente y el volumen de agua contaminada dentro del reactor fue de $100 \mathrm{~L}$. Por otro lado, en los ejemplos 3 y 4 el volumen del reactor fue de $80 \mathrm{~L}$ y la concentración de descarga se fijó en 12 y $10 \mathrm{mg} \mathrm{L}^{-1}$, respectivamente. En todos los ejemplos se utilizó como base los datos de la Tabla 1 y la superestructura básica de la Fig. 1.

Ejemplo $1\left(c_{j, e} e^{=}=12 \mathrm{mgL}^{-1}\right.$ y $\left.V=100 \mathrm{~L}\right)$

Los resultados obtenidos en este caso se muestran en la Tabla 2. El diseño final para la red de tratamiento se presenta en la Fig. 2. Las corrientes 1,2 y una fracción del caudal 3 fueron tratados en el reactor electroquímico, mientras que los flujos 4, 5 y la fracción no tratada de caudal 3 se llevaron directamente al mezclador 2. El caudal de agua tratada para este caso es $12.2 \mathrm{~L} \mathrm{~min}^{-1}$, es decir, $53 \%$ del caudal total.

Tabla 2: Solución óptima del Ejemplo 1, con $\mathrm{C}_{\mathrm{j}, \mathrm{e}} \mathrm{U}=12 \mathrm{mgL}^{-1}$ y $\mathrm{V}=100 \mathrm{~L}$

\begin{tabular}{|c|c|}
\hline $\mathrm{t}\left(\mathrm{L} \mathrm{min}^{-1}\right)$ & 12.2 \\
\hline $\operatorname{cin}_{\mathrm{j}}\left(\mathrm{mg} \mathrm{L}^{-1}\right)$ & 24.3 \\
\hline $\operatorname{cout}_{\mathrm{j}}\left(\mathrm{mg} \mathrm{L}^{-1}\right)$ & 7.234 \\
\hline $\mathrm{C}_{\mathrm{j}, \mathrm{e}}\left(\mathrm{mg} \mathrm{L}^{-1}\right)$ & 12 \\
\hline$\Delta \mathrm{m}_{\mathrm{j}}\left(\mathrm{g} \mathrm{min}^{-1}\right)$ & 0.21 \\
\hline $\mathrm{R}_{\mathrm{j}}$ & 0.7 \\
\hline $\mathrm{V}(\mathrm{L})$ & 100 \\
\hline
\end{tabular}

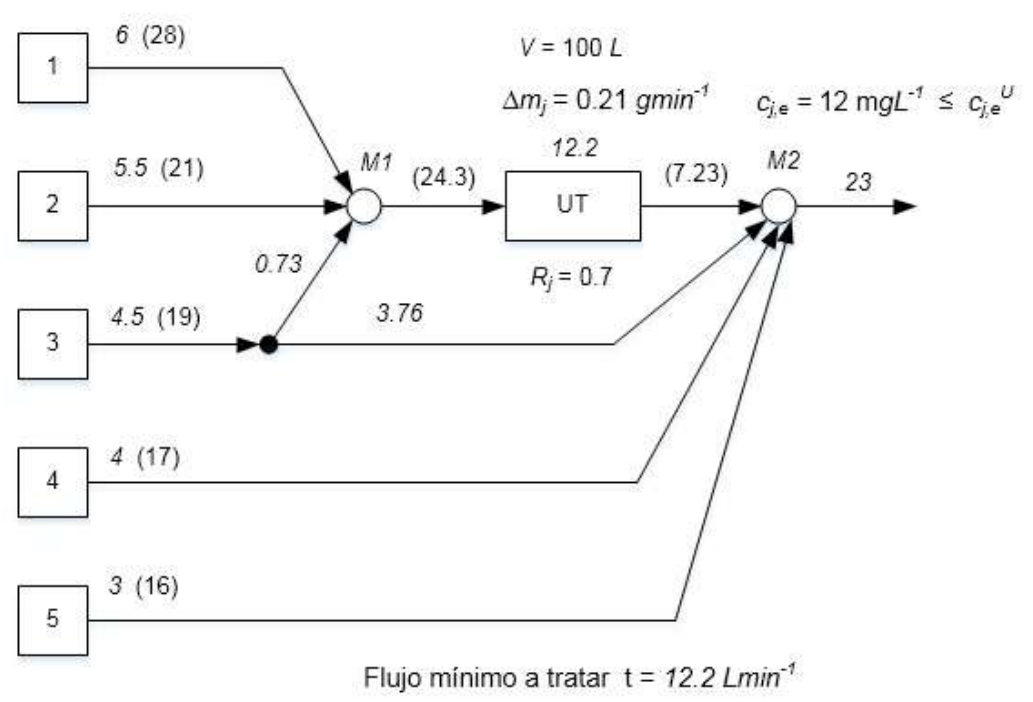

Fig. 2: Diseño óptimo para la red de tratamiento del Ejemplo 1 (los flujos de corrientes (Lmin ${ }^{-1}$ ) se presentan en itálicas y las concentraciones $\left(\mathrm{mgL}^{-1}\right)$ se presentan entre paréntesis). 
Ejemplo $2\left(c_{j, e}=10 \mathrm{mgL}^{-1}\right.$ y $\left.V=100 \mathrm{~L}\right)$

Los resultados obtenidos en este caso se muestran en la Tabla 3. El diseño final para la red de tratamiento de aguas residuales se presenta en la Fig. 3. Las corrientes 1, 2, 3 y una fracción del caudal 4 fueron tratados en el reactor electroquímico, mientras que el flujo 5 y la fracción no tratada del caudal 4 se llevaron directamente al mezclador 2 . El caudal de agua tratada para este caso es $18.4 \mathrm{~L} \mathrm{~min}^{-1}$, es decir, $80 \%$ del caudal total. De acuerdo con estos resultados, al aumentar la carga másica de amoxicilina a remover de 0.21 a $0.26 \mathrm{~g} \mathrm{~min}^{-1}$ el porcentaje de caudal tratado aumenta un $27 \%$ (de $12.2 \mathrm{~L} \mathrm{~min}^{-1}$ a $18.4 \mathrm{~L} \mathrm{~min}^{-1}$ ).

Tabla 3: Solución óptima del Ejemplo 2, con $\mathrm{C}_{\mathrm{j}, \mathrm{e}} \mathrm{U}=10 \mathrm{mgL}^{-1}$ y $\mathrm{V}=100 \mathrm{~L}$.

\begin{tabular}{|c|c|}
\hline $\mathrm{t}\left(\mathrm{Lmin}^{-1}\right)$ & 18.4 \\
\hline $\operatorname{cin}_{\mathrm{j}}\left(\mathrm{mg} \mathrm{L}^{-1}\right)$ & 22.3 \\
\hline $\operatorname{cout}_{\mathrm{j}}\left(\mathrm{mg} \mathrm{L}^{-1}\right)$ & 8.409 \\
\hline $\mathrm{C}_{\mathrm{j}, \mathrm{e}}\left(\mathrm{mg} \mathrm{L}^{-1}\right)$ & 10 \\
\hline$\Delta \mathrm{m}_{\mathrm{j}}\left(\mathrm{g} \mathrm{min}^{-1}\right)$ & 0.26 \\
\hline $\mathrm{R}_{\mathrm{j}}$ & 0.62 \\
\hline $\mathrm{V}(\mathrm{L})$ & 100 \\
\hline
\end{tabular}

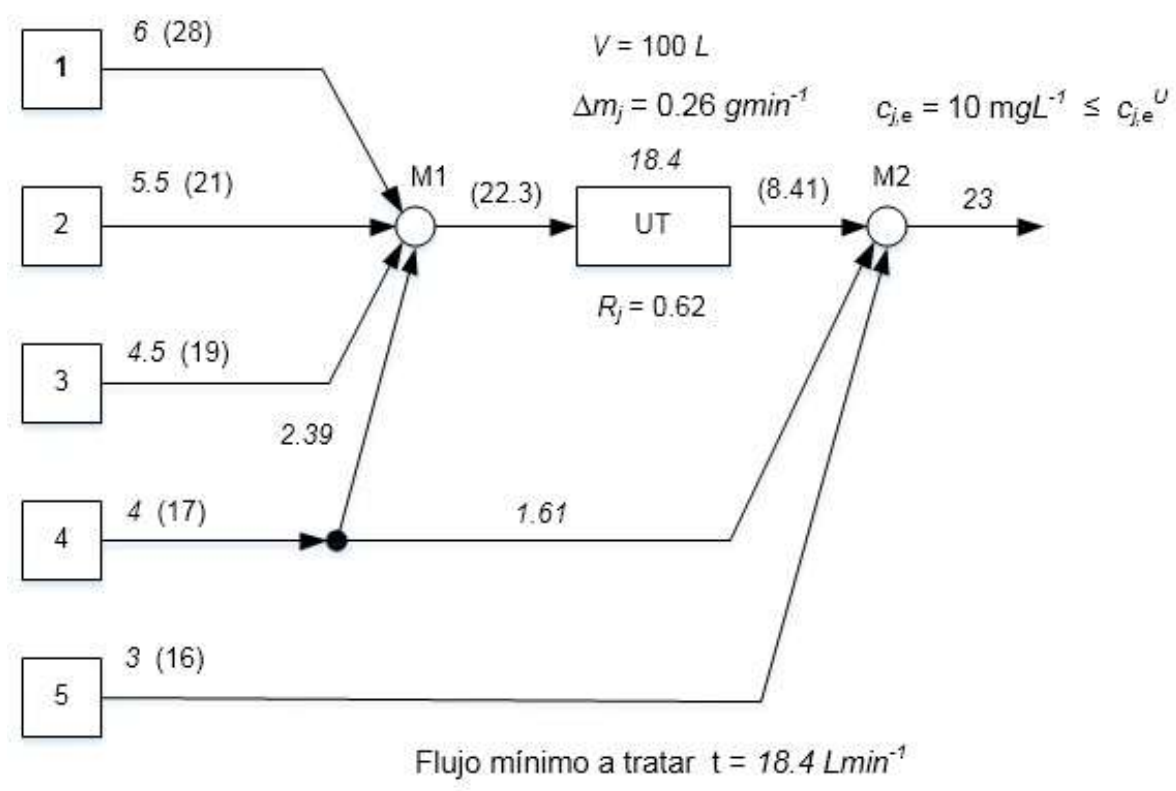

Fig. 3: Diseño óptimo para la red de tratamiento del Ejemplo 2 (los flujos de corrientes ( Lmin $\left.^{-1}\right)$ se presentan en itálicas y las concentraciones $\left(\mathrm{mgL}^{-1}\right)$ se presentan entre paréntesis).

Ejemplo $3\left(c_{j, e} \mathrm{U}=12 \mathrm{mgL}^{-1}\right.$ y $\left.\mathrm{V}=80 \mathrm{~L}\right)$

Los resultados obtenidos se muestran en la Tabla 4. El diseño final para la red de tratamiento de aguas residuales se presenta en la Fig. 4. Las corrientes 1, 2 y una fracción del caudal 3 fueron tratados en el reactor electroquímico, mientras que los flujos 4, 5 y la fracción no tratada del caudal 3 se enviaron directamente al mezclador 2. El caudal de agua tratada para este caso es $13.8 \mathrm{~L} \mathrm{~min}^{-1}$, aproximadamente el $55 \%$ del caudal total.

Tabla 4: Solución óptima del Ejemplo 3, con $\mathrm{C}_{\mathrm{j}, \mathrm{e}} \mathrm{U}=12 \mathrm{mgL}^{-1}$ y $\mathrm{V}=80 \mathrm{~L}$.

\begin{tabular}{|c|c|}
\hline$t\left(\mathrm{~L} \mathrm{~min}^{-1}\right)$ & 13.8 \\
\hline $\operatorname{cin}_{\mathrm{j}}\left(\mathrm{mg} \mathrm{L}^{-1}\right)$ & 23.71 \\
\hline $\operatorname{cout}_{\mathrm{j}}\left(\mathrm{mg} \mathrm{L}^{-1}\right)$ & 8.56 \\
\hline $\mathrm{C}_{\mathrm{j}, \mathrm{e}}\left(\mathrm{mg} \mathrm{L}^{-1}\right)$ & 12 \\
\hline$\Delta \mathrm{m}_{\mathrm{j}}\left(\mathrm{g} \mathrm{min}^{-1}\right)$ & 0.21 \\
\hline $\mathrm{R}_{\mathrm{j}}$ & 0.64 \\
\hline $\mathrm{V}(\mathrm{L})$ & 80 \\
\hline
\end{tabular}




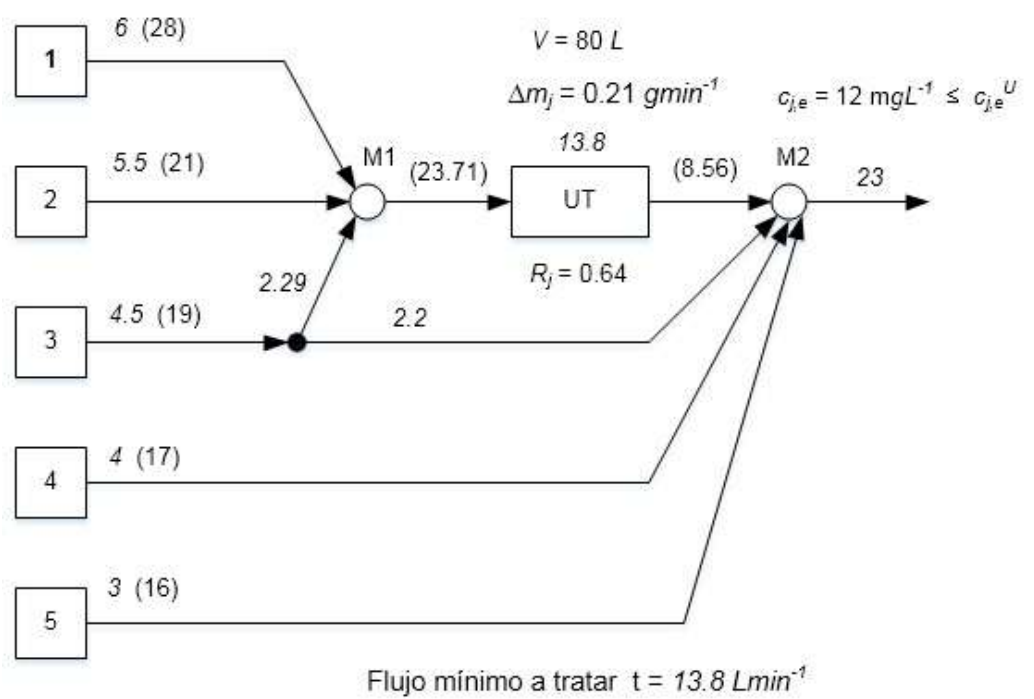

Fig. 4: Diseño óptimo para la red de tratamiento del Ejemplo 3 (los flujos de corrientes (Lmin ${ }^{-1}$ ) se presentan en itálicas y las concentraciones $\left(\mathrm{mgL}^{-1}\right)$ se presentan entre paréntesis).

Ejemplo $4\left(c_{j, e} e^{-10} \mathrm{mgL}^{-1}\right.$ y $\left.V=80 \mathrm{~L}\right)$

Los resultados obtenidos en este caso se muestran en la Tabla 5. El diseño final para la red de tratamiento de aguas residuales se presenta en la Fig. 5. Las corrientes 1, 2, 3, 4 y una fracción del caudal 5 fueron tratados en el reactor electroquímico, mientras que la fracción no tratada del caudal 5 se envió directamente al mezclador 2. El caudal de agua tratada para este caso es $22.5 \mathrm{~L} \mathrm{~min}-1$, i.e., se trató casi el caudal total. De acuerdo con estos resultados, al aumentar la carga másica de amoxicilina a remover de 0.21 a 0.26 gmin $^{-1}$ el caudal tratado aumenta considerablemente, esto también está relacionado con la disminución en el volumen del reactor con el consiguiente aumento en los costos del tratamiento.

Tabla 5: Solución óptima del Ejemplo 4, con $\mathrm{C}_{\mathrm{j}, \mathrm{e}} \mathrm{U}=10 \mathrm{mgL}^{-1}$ y V $=80 \mathrm{~L}$.

\begin{tabular}{|c|c|}
\hline $\mathrm{t}\left(\mathrm{L} \mathrm{min}^{-1}\right)$ & 22.5 \\
\hline $\operatorname{cin}_{\mathrm{j}}\left(\mathrm{mg} \mathrm{L}^{-1}\right)$ & 21.2 \\
\hline $\operatorname{cout}_{\mathrm{j}}\left(\mathrm{mg} \mathrm{L}^{-1}\right)$ & 9.865 \\
\hline $\mathrm{C}_{\mathrm{j}, \mathrm{e}}\left(\mathrm{mg} \mathrm{L}^{-1}\right)$ & 10 \\
\hline$\Delta \mathrm{m}_{\mathrm{j}}\left(\mathrm{g} \mathrm{min}^{-1}\right)$ & 0.26 \\
\hline $\mathrm{R}_{\mathrm{j}}$ & 0.54 \\
\hline $\mathrm{V}(\mathrm{L})$ & 80 \\
\hline
\end{tabular}

Los resultados de los cuatro ejemplos se resumen en la Tabla 6 y muestran el comportamiento del sistema cuando se modifica el volumen del reactor y la concentración de amoxicilina en la descarga. Para los ejemplos 1 y 2 se puede observar que al reducir el parámetro de concentración límite permitida en el punto de descarga $\left(c_{j, e}\right.$ y) y manteniendo constante el volumen del reactor $(V)$, el flujo másico $\left(\Delta m_{j}\right)$ de contaminante a remover aumenta y por ende también el caudal de tratamiento $(t)$, sin embargo, la tasa de remoción $\left(R_{j}\right)$ disminuye debido a que la concentración de entrada es menor y se tiene una mayor dilución. Es decir, se demanda un mayor tratamiento de efluentes para el ejemplo 2.

Tabla 6: Resultados de diseño de red de tratamiento para los ejemplos.

\begin{tabular}{|c|c|c|c|c|c|c|c|}
\hline Ejemplo & $\begin{array}{l}\text { V } \\
(\mathrm{L})\end{array}$ & $\begin{array}{c}\mathrm{C}_{\mathrm{j}, \mathrm{e}} \mathrm{U} \\
\left(\mathrm{mg} \mathrm{L}^{-1}\right)\end{array}$ & $\begin{array}{c}\mathrm{t} \\
\left(\mathrm{L} \mathrm{min}^{-1}\right)\end{array}$ & $\begin{array}{c}\operatorname{cin}_{j} \\
\left(m g L^{-1}\right)\end{array}$ & $\begin{array}{c}\text { cout }_{j} \\
\left(\mathrm{mg} \mathrm{L}^{-1}\right)\end{array}$ & $\mathrm{R}_{\mathrm{j}}$ & $\begin{array}{c}\Delta \mathrm{m}_{\mathrm{j}} \\
\left(\mathrm{g} \mathrm{min}^{-1}\right)\end{array}$ \\
\hline 1 & \multirow{2}{*}{100} & 12 & 12.239 & 24.30 & 7.234 & 0.70 & 0.21 \\
\hline 2 & & 10 & 18.392 & 22.30 & 8.409 & 0.62 & 0.26 \\
\hline 3 & \multirow{2}{*}{80} & 12 & 13.794 & 23.71 & 8.560 & 0.64 & 0.21 \\
\hline 4 & & 10 & 22.495 & 21.20 & 9.865 & 0.54 & 0.26 \\
\hline
\end{tabular}




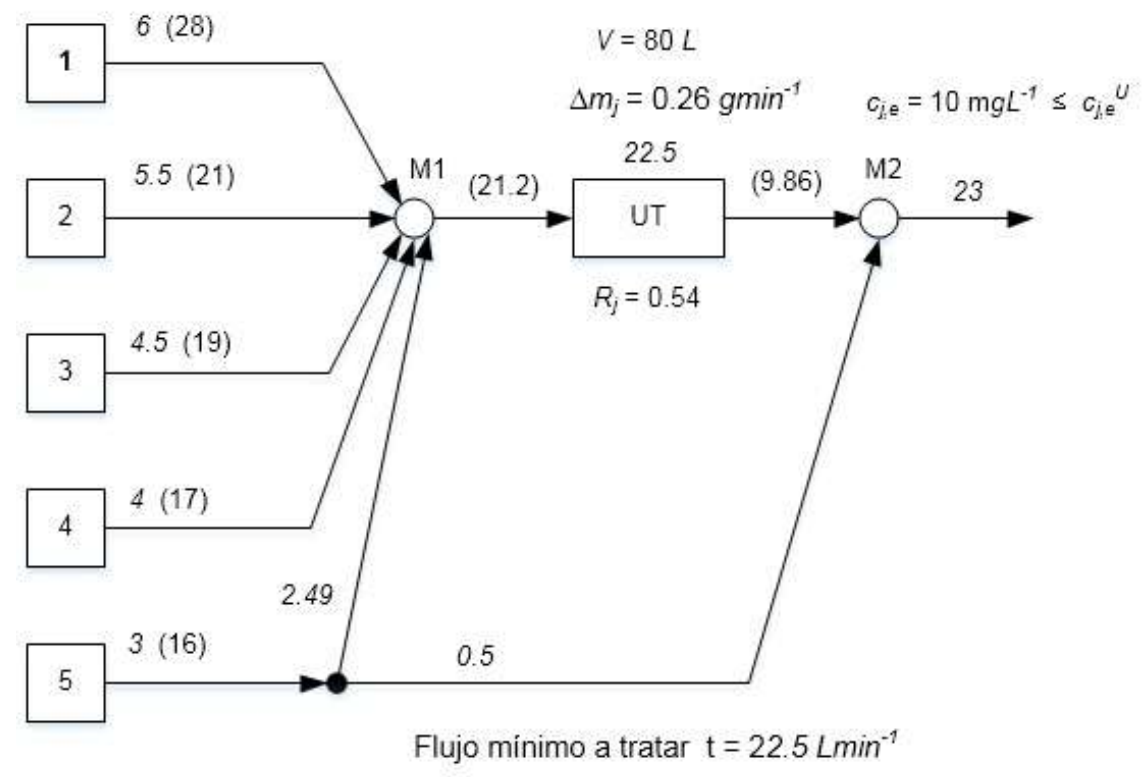

Fig. 5: Diseño óptimo para la red de tratamiento del Ejemplo 4 (los flujos de corrientes (Lmin $\left.{ }^{-1}\right)$ se presentan en itálicas y las concentraciones $\left(\mathrm{mgL}^{-1}\right)$ se presentan entre paréntesis).

Por otro lado, si se comparan los resultados de los ejemplos 1 y 3 , en donde el volumen disminuye y la concentración limite se mantiene constante, la tasa de remoción también disminuye debido a que el caudal de tratamiento aumenta y la concentración a la entrada del reactor es menor, finalmente el flujo másico de contaminante a remover se mantiene constante ya que el objetivo es remover una cantidad precisa de contaminante.

\section{CONCLUSIONES}

Los contaminantes emergentes como lo son algunos antibióticos, están surgiendo como una de las principales alertas ambientales. Los trabajos que se han realizado recientemente han señalado el uso de algunos métodos de electroquímica como viables para la eliminación de estos contaminantes, entre ellos la amoxicilina. Los resultados para los cuatro ejemplos de diseño óptimo presentados en este trabajo, muestran que el enfoque de programación matemática es relevante y de fácil implementación cuando las ecuaciones de balance de materia o de diseño exhiben formulaciones complejas, como en el caso de un proceso de tratamiento por electrocoagulación. El modelo de programación no lineal desarrollado permite obtener el diseño de un sistema de eliminación de amoxicilina mediante la minimización del caudal utilizando una superestructura básica a fin de incluir un conjunto solución amplio. Se determinaron condiciones óptimas para el sistema de tratamiento de cada uno de los cuatro ejemplos abordados. Se encontró que, el uso de reactores con menor volumen y una restricción más rigurosa para el contaminante en el punto de descarga, promueven un tratamiento total y una mayor dilución de efluentes, lo cual se traduce en un mayor costo de tratamiento.

\section{NOTACIÓN}

$\begin{array}{ll}\text { UT } & \text { Unidad de tratamiento } \\ \text { M1 } & \text { Mezclador } 1 \\ \text { M2 } & \text { Mezclador } 2 \\ \text { Índices } & \\ i & \text { Corrientes } \\ j & \text { Contaminante-amoxicilina } \\ \mathrm{e} & \text { Punto de descarga } \\ \mathrm{U} & \text { Límite superior } \\ \mathrm{L} & \text { Límite inferior }\end{array}$

Conjuntos

$I=\{i: i\} \quad$ Conjunto de corrientes de agua residual 


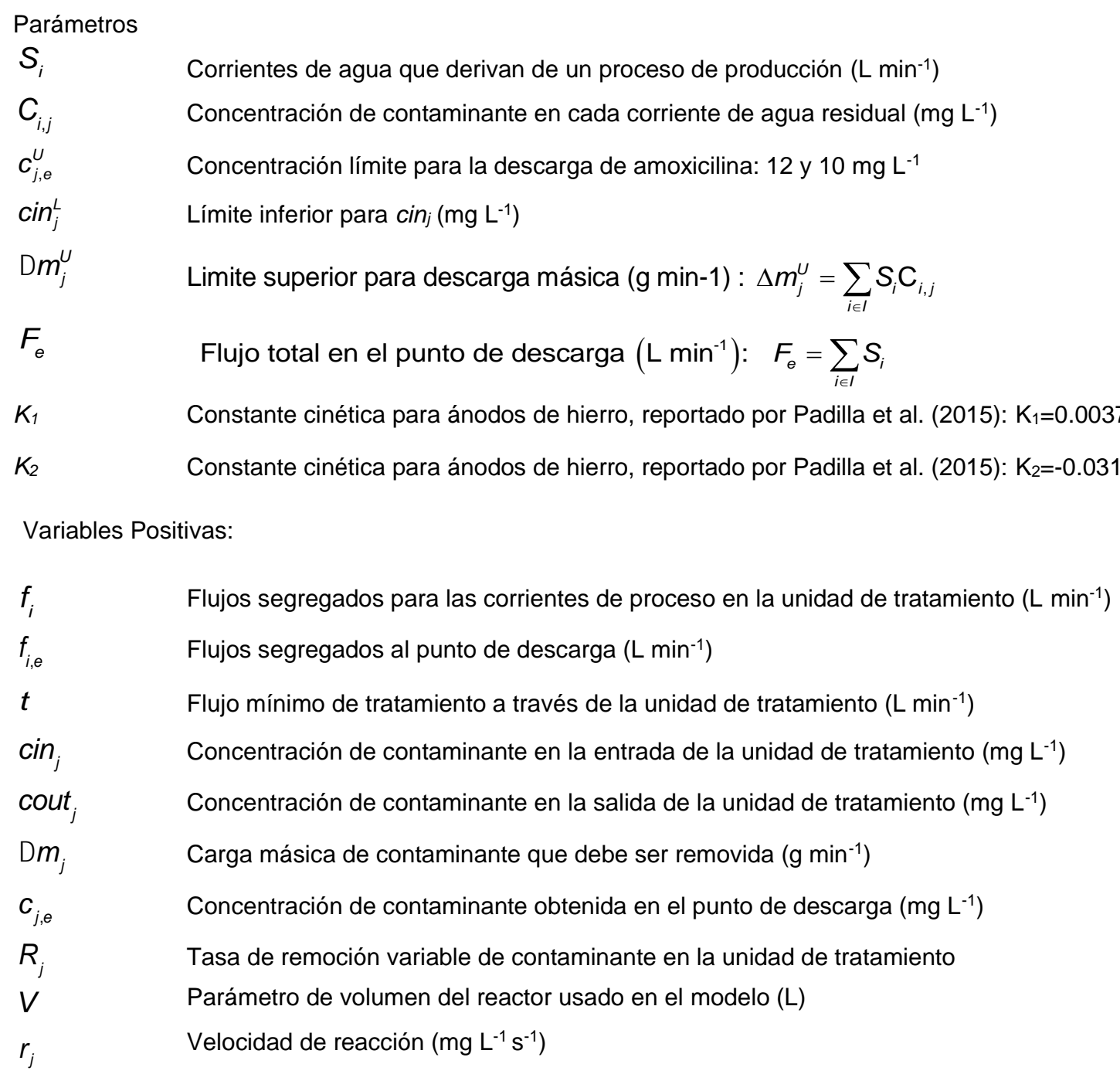

\section{REFERENCIAS}

Alva, A.; A. Kokossis y R. Smith, The Design of Water-Using Systems in Petroleum Refining Using a Waterpinch Decomposition, Chemical Engineering Journal, 128(1), 33-46 (2007)

Arsand, D.R.; K. Kümmerer y A.F. Martins, Removal of Dexamethasone from Aqueous Solution and Hospital Wastewater by Electrocoagulation, Science of the Total Environment, 443, 351-357 (2013)

Bagajewicz, M., A Review of Recent Design Procedures for Water Networks in Refineries and Process Plants, Computers \& Chemical Engineering, 24(9-10), 2093-2113 (2000)

Brooke, A.; D. Kendrick; A. Meeraus y R. Raman, GAMS: A User's Guide. GAMS Development Corporation, Estados Unidos de América (2012)

Butler, E.; Y.-T. Hung; R.Y.-L. Yeh y M. S. Al Ahmad, Electrocoagulation in Wastewater Treatment. Water, 3(2), 495-525 (2011)

Dreser, A., V. J. Wirtz, K. K. Corbett, G. Echániz, Uso de antibióticos en México: revisión de problemas y políticas, Salud Pública de México, ISSN: 1606-7916 (en línea), 50(3), S480-S487, 2008. http://www.scielo.org.mx/pdf/spm/v50s4/09.pdf. Acceso: 11 de Febrero (2015)

Faria, D. y M. Bagajewicz, Profit-based Grassroots Design and Retrofit of Water Networks in Process Plants, Computers and Chemical Engineering, 33(2), 436-453 (2009)

Fent, K.; A.A. Weston y D. Caminada, Ecotoxicology of Human Pharmaceuticals, Aquatic Toxicology, 76(2), 122-159 (2006) 
Freitas, T., V. Oliveira, M. de Souza, H. Geraldino, V. Almeida, S. Fávaro y J. Garcia, Optimization of coagulation-flocculation process for treatment of industrial textile wastewater using okra (A. esculentus) mucilage as natural coagulant, Industrial Crops and Products, 76, 538-544 (2015)

Galán, B. y I.E. Grossmann, Optimization Strategies for the Design and Synthesis of Distributed Wastewater Treatment Networks, Computers \& Chemical Engineering, 23, S161-S164 (1999)

García, C, Entérate: Antibióticos que más se venden en México. El Universal, Compañía Periodística Nacional, 2010. http://www.eluniversal.com.mx/notas/668756.html. Acceso: 11 de Febrero (2015)

Grossmann, I.E.; J.A. Caballero y H. Yeomans, Mathematical Programming Approaches to the Synthesis of Chemical Process Systems, Korean Journal of Chemical Engineering, 16(4), 407-426 (1999)

Hernández-Suárez, R.; J. Castellanos-Fernández y J.M. Zamora, Superstructure Decomposition and Parametric Optimization Approach for the Synthesis of Distributed Wastewater Treatment Networks, Ind. Eng. Chem. Res., 43(9), 2175-2191 (2004)

Homem, V.; A. Alves y L. Santos, Amoxicillin Degradation at ppb Levels by Fenton Oxidation Using Design of Experiments, Science of the Total Environment, 408(24), 6272- 6280 (2010)

Klavarioti, M.; D. Mantzavinos y D. Kassinos, Removal of Residual Pharmaceuticals from Aqueous Systems by Advanced Oxidation Processes, Environment international, 35(2), 402-417 (2009)

Nidheesh, P.V. y R. Gandhimathi, Trends in Electro-Fenton Process for Water and Wastewater Treatment: an Overview, Desalination, 299, 1-15 (2012)

Padilla, B.G.; A. Alonso; S.A. Martínez; M. González; U.J. Jaúregui y J. Ramírez, Electrochemical Degradation of Amoxicillin in Aqueous Media, Chem. Eng. and Processing: Process Intensification, 94, 93-98 (2015)

Petrovic, M.; S. Gonzalez y D. Barceló, Analysis and Removal of Emerging Contaminants in Wastewater and Drinking Water, TrAC Trends in Analytical Chemistry, 22(10), 685-696 (2003)

Quesada, I.; C. Julcour; U.J. Jáuregui; A.M. Wilhelm y H. Delmas, Sonolysis of Levodopa and Paracetamol in Aqueous Solutions, Ultrasonics Sonochemistry, 16(5), 610-616 (2009)

Wang, Y.P. y R. Smith, Design of Distributed Effluent Treatment Systems, Chemical Engineering Science, 49(18), 3127-3145 (1994)

Xuedong Z., H. Jianmei, H. Spanjers, J. van Lier, Performance of inorganic coagulants in treatment of backwash waters from a brackish aquaculture recirculation system and digestibility of salty sludge, Aquacultural Engineering, 61, 9-16 (2014)

Zamora-Mata, J.M.; R. Hernández-Suárez y J. Castellanos-Fernández, Modelo de Programación Lineal para Asistir en el Diseño de Sistemas Distribuidos de Tratamiento de Efluentes, Revista Mexicana de Ingeniería Química, 3(1), 121-134 (2004) 
